\title{
COIFLET-BASED FUZZY-CLASSIFIER FOR DEFECT DETECTION IN INDUSTRIAL LNG/LPG TANKS
}

\author{
Uvais Qidwai and Mohamed Shakir \\ KINDI Research Center, Department of Computer Science \& Engineering, \\ Qatar University, Doha, Qatar \\ uqidwai@qu.edu.qa, shakir@qu.edu.qa
}

\begin{abstract}
This paper describes a classification method for raw sensor data using a Fuzzy Inference System to detect the defects in large LNG tanks. The data is obtained from a Magnetic Flux Leakage (MFL) sensing system which is usually used in the industry to located defects in metallic surfaces, such as tank floors. A robotic inspection system has been developed in conjunction with the presented work which performs the same inspection tasks at much lower temperatures than human operators would thus reducing the shutdown time significantly which is typically of the order of 15-20 million Dollars per day. The main challenge was to come up with an algorithm that can map the human heuristics used by the MFL inspectors in field to locate the defects into an automated system and yet keep the algorithm simple enough to be deployed in near real-time applications. Unlike the human operation of the MFL equipment, the proposed technique is not very sensitive to the sensor distance from the test surface and the calibration requirements are also very minimal which are usually a big impediment in speedy inspections of the floor by human operator. The use of wavelet decomposition with Coiflet waves has been utilized here for deconvolving the essential features of the signal before calculating the classification features. This wavelet was selected to its canny resemblance with the actual MFL signals that makes these wavelets very natural basis function for decomposition..
\end{abstract}

\section{KEYWORDS}

Nondestructive Testing (NDT), Fuzzy Inference System, Defect Detection, Classification, filters, Wavelet based Deconvolution, Coiflet Transform.

\section{INTRODUCTION}

Liquefied Natural-Petroleum Gas (LNG), Liquefied Petroleum Gas (LPG) and Field/Plant Condensate are some of the main products of oil and gas sector in the Middle East and phenomenal revenue is associated with their trade. Both LNG and LPG are usually stored at very low temperatures which pose a major issue in maintaining the tank since the tank must be shut down for a period of 10-14 days before the tanks can be safe for human ingress. The maintenance cost and time for such an operation can be significantly reduced if the shutdown period can be decreased. One of the ways that this can be achieved is by deploying a specially designed robot, capable of working at very low temperatures and in hazardous conditions, thus, replacing the need for risking human personnel during initial stages of inspection and at the same time reducing the shutdown cost. Such robotic system is capable of inspecting the floor which sits on a solid

Dhinaharan Nagamalai et al. (Eds) : CSE, DBDM, CCNET, AIFL, SCOM, CICS, CSIP- 2014 pp. 197-207, 2014. (C) CS \& IT-CSCP 2014

DOI : $10.5121 /$ csit.2014.4417 
concrete flooring and is virtually impossible to be accessed from outside $[1,2]$. The presented technique is in conjunction with the design of one such robot and its application in real industrial environment.

Robotics is an extension of human abilities and can create a safety environment for work where needed. Robots can, through adaptive design, perform tele-operation and act as smart tools, handle an assortment of tasks while communicating and interacting with workers located on-site or remotely. This supports sustainable production at the cutting-edge of efficiency and creativity. Remotely Operated Vehicles (ROVs) have become an important tool in many industrial and environmental applications. On one hand, such robots reduce the hazardous exposures of the human personnel by operating in such dangerous or harsh environments, and at the same time they increase the level of accuracy in measurements and inspections by not being limited with the human weaknesses and subjectiveness. The developed robotic system is exactly based around this idea. It can operate at very low temperature where human exposure will be too dangerous as well as too expensive. At the same time, the intelligent Fuzzy classification algorithm accumulates the experiences from several experts from the industry and is not limited by human weaknesses. By using this intelligent system in the robotic platform, the tank floor can be inspected remotely as well as autonomously with more accurate detection.

Large chemical and petroleum product storage tanks are mostly made of steel plates that are welded together to form the structure. The material and the welds are inspected for manufacturing defects when constructed but must also be periodically inspected throughout their service life for signs of damage. The carbon steel is prone to attack by corrosion and in some circumstances cracks can form over time. NDT personnel use visual, X-ray, ultrasonic and other inspection methods to search for flaws and service-induced damage.

\section{EXPERIMENTAL SETUP}

The MFL inspection system used in this work is quite standard in the industry for this application. The detection the defects in the floor of the tank are sensed by used MFL sensor using the theory of Hall Effect sensing. Magnetic Flux Leakage (MFL) is a phenomenon in which the magnetic flux escapes out of the normal magnetic path due to disruptions in the path or broken paths. After ferromagnetic materials are magnetized, the leakage magnetic field can be created above its surface because of its internal defects, the method to measure leakage magnetic field by sensors is called magnetic flux leakage testing (MFL) [3-10]. Figure 1 shows the robot used in this work.

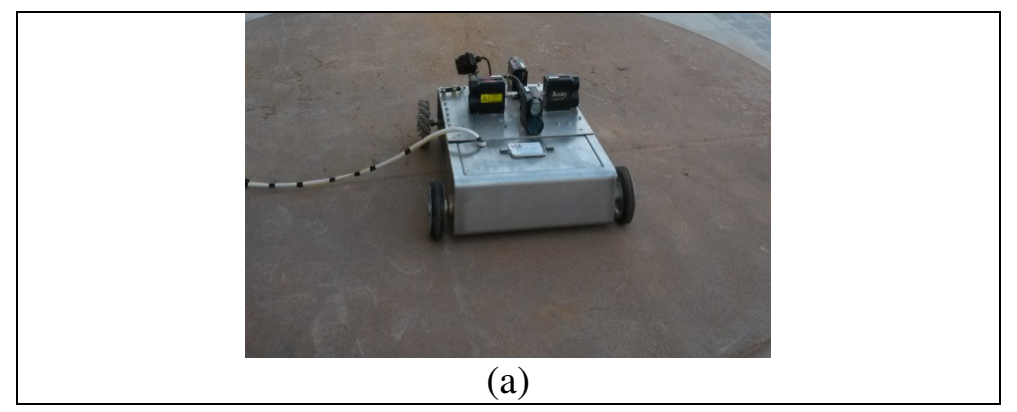




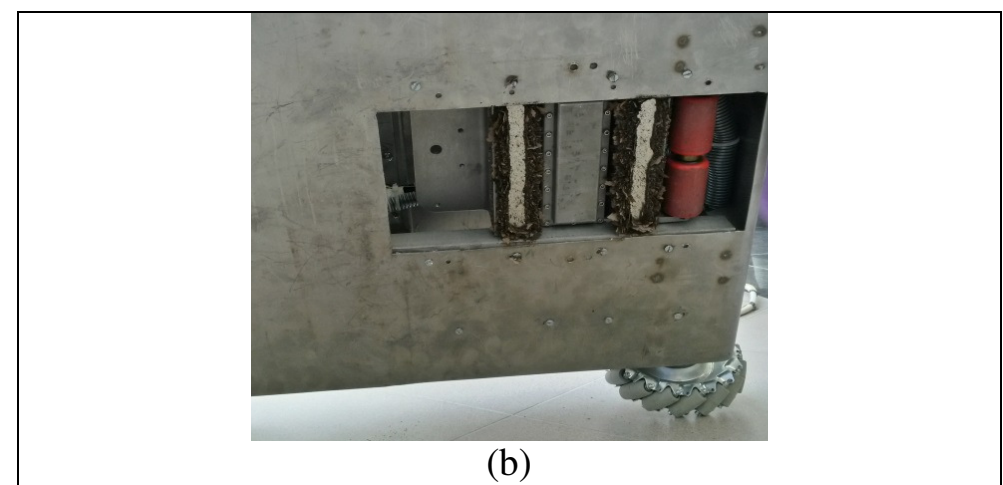

Figure 1. The robotic system used in this work for data collection. (a) The inspection robot on a test plate, and (b) the MFL sensor fixed under the robot's body.

The MFL Data is an exciting source for a variety of signal processing techniques and researchers have been quite busy in applying all sorts of conventional and non-conventional approaches in identifying the defects from the data. Techniques like Wavelet Transforms, Genetic Algorithms, Particle Swarm Optimization, and other well-known filtering techniques have been applied on such data [11-15]. Each technique has its own merit in terms of the application. However, all of these do have a large computational time requirements. In this work, the main focus was on developing a technique that could be utilized in near-real-time scenarios for active inspections using the robotic platform.

\section{AlgORITHM}

As the robot moves across the testing area, the MFL data is collected, standard signal processing techniques are applied on it. Primarily this corresponds to normalization and low-pass filtering for noise removal. Based on the shapes of the MFL data waveforms, following strategy was adapted:

1. Coiflet wavelet $[16,17]$ was selected based on its similarity in shape with the MFL waveform. Coiflets are discrete wavelets designed by Ingrid Daubechies, at the request of Ronald Coifman, to have scaling functions with vanishing moments.

2. Comparing with the actual MFL waveform, this wavelet must be smoothed and skewed. This was done using convolution with a Gaussian wavelet. The one dimensional Gaussian wavelet has an impulse response given by:

$$
g(x)=\sqrt{\frac{a}{\pi}} e^{-a x^{2}}
$$

Where $\mathrm{x}$ is the distance from the origin in the horizontal axis and $\sigma$ is the standard deviation of the Gaussian distribution. A typical coiflet generated as part of this work is shown in Figure 2 along with the actual Coiflet mother wavelet. 


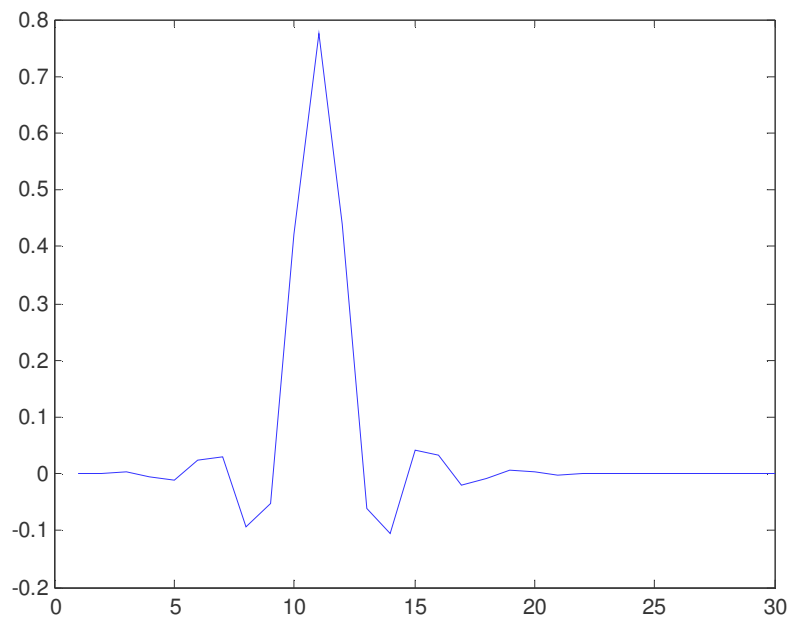

Figure 2. Coiflet mother wavelet used in this work for decomposing the MFL signals into low and high frequency components.

By using Coiflet-based deconvolution, removing all the noise and base signal artifact from the raw MFL signal is achived. This is done by:

- Decomposing the raw signal into its Approximation and Detail components [CA and CD].

- Then the CA is decomposed again into the respective components [CAA and CDD].

- These two decompositions have been found to be sufficient in removing all the unwanted sensor noise, metallic approximations, and quantization artifacts from the raw signal.

- Then, an inverse transform is applied in order to regenerate the original signal by combining the two approximation components (CAA and CA) with corresponding detail components reset to zero.

This results in a cleaner signal which can be better analyzed for the subsequent classification step. Figure 3 shows the result of deconvolution on the raw MFL signal.

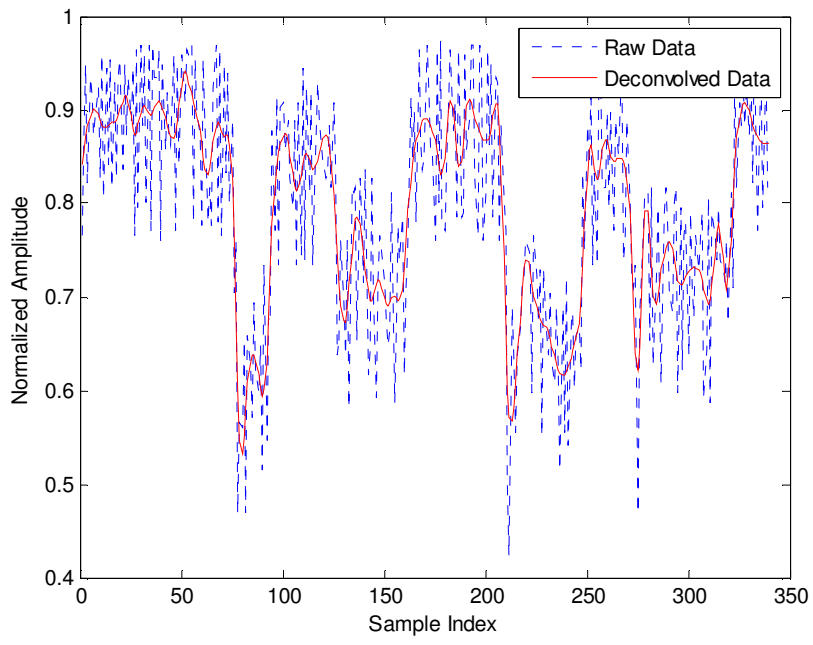

Figure 3. Deconvlution result on the MFL signal. The dotted blue line shows the Raw MFL data and the solid red line shows the deconvolved signal. 
Figure 4 represents the complete Algorithm, including the subsequent steps of Feature Calculation and Fuzzy Inference System, which are further elaborated in the following sections.

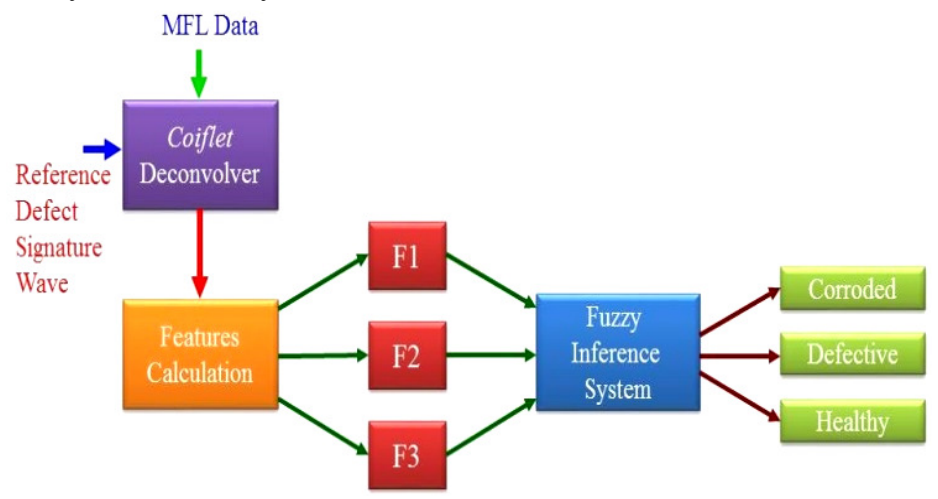

Figure 4. Main signal processing algorithm applied on the raw MFL data.

The algorithm is applied on a finite length of the stored or a window of on-the-fly data from the robot's sensors and correlates it to the points on the floor map of the tank. For each window of samples taken for analysis, the algorithm results into the classified areas of healthy, corroded, and defective states. For the possible defective states, recommendation is made for the maintenance staff to look more carefully into the possibility of having a further classification. The first 100 Coiflet coefficients, the feature set is also stored in a simple database in order to be used later on for more detailed classification.

\section{THE FuZZY INFERENCE SYSTEM}

The actual understanding of the waveforms is obtained by the program through a set of measurable values, called Features. In order to select a meaningful feature set, human heuristics were heavily utilized in this work. By quantifying the human perception according to the general understanding of the information present in the measurements by the NDT expert, a better classification is obtained that will assist the technicians to make a better decision concerning maintenance, material selection and replacement. Fuzzy Inference System is probably the most suitable tool for this purpose. While it is a wonderful tool to map the imprecise or heuristically oriented information into quantitative data, it also maps the human decision making capabilities using a subjective Rule base. This may raise some issues related to the accuracy since it is based on less precise information and the usefulness of the system on the whole may be questioned. Actually, when limitations of NDT personnel in the field are considered, these are considered subjectively. This means that the accuracy of the NDT process is limited by expertise of a single individual. This is supplemented further with the classical limitations associated with human operators such as tiredness, physical and mental uprightness, and proper understanding of the problem. In order to automate this process, the heuristical perceptions of the human operators must be quantified into some measureable parameters (or features). The Features selected for the work presented in this work are, therefore, based on translating the human understanding into the mathematical quantities. In the real practice, the NDT expert will use the MFL scanning system and drag it over the test surface. An audio signal (beep) is the target outcome from these experiments. The degree amplitude and durations of these beeps are directly related to the depth and width of the defect under the sensor head, respectively. Once a signal is heard, the place is marked with spray-paint for more careful follow-up inspections. These characteristics of the testing procedure are captured in this work by using two sources: 
1. Human heuristics from NDT experience, and

2. Deconvolved signals' statistical features.

In order to understand the mechanism of how a human expert would classify the metal health using MFL data, several experts were consulted with including experts from the top manufacturer of the MFL sensing equipment used in the paper, Silver Wing UK. Based on the collective experiences of all of these experts, the codes defined in the MFL based inspections, and the way the MFL sensors are used by the human operators, following heuristical features can be deduced as indicators of various health parameters of the sample:

- Big change in the MFL signal representing a deep flaw.

- Long voids (amplitude changes) in the MFL signal represents wider surface defects.

- Grass like; low-amplitude signals represent healthy metal.

- Corrosion is defined by several modes of the grass signals that fall between the deep defects and the healthy metal.

Based on the human heuristical parameters, following features were devised to be calculated from the raw and deconvolved signals:

F1. AWR (Amplitude to Width ratio) of the big change in the MFL signal (deconvolved signal only)

F2. GE (Grass Energy) is the sum squared values of the grass signal below $10 \%$ of the maximum value in the MFL signal (Raw signal only)

F3. BE (Bush Energy) is the sum squared values of the grass-like signal between $10 \%$ and $30 \%$ of the maximum value in the MFL signal (Deconvolved signal only).

Using these features, following Membership functions were made to map the actual values from the data into the Fuzzy space (fuzzification of data). The overall Fuzzy classification system is shown in Figure 5.

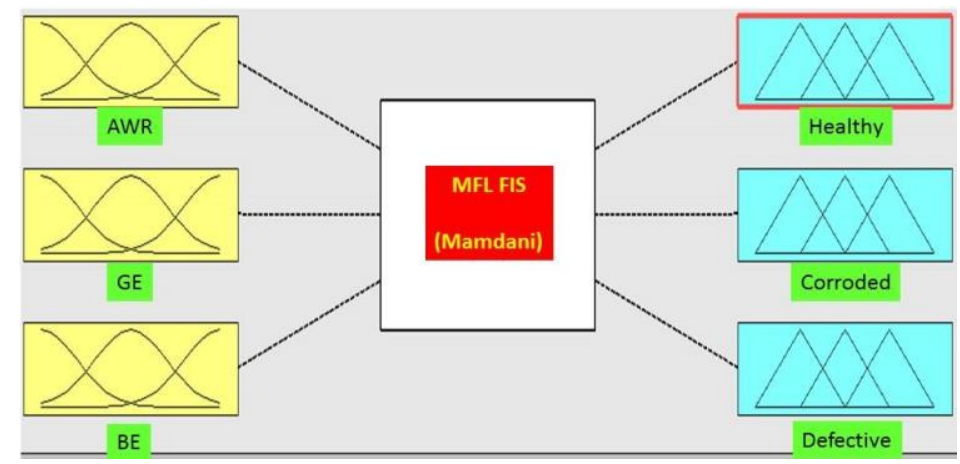

Figure 5. Fuzzy Inference System for classifying the input features (AWR, GE, and BE) into one of the output classes (Healthy, Corroded, Defective).

The input features are calculated on the fly as the data is obtained and are then fuzzified by the input membership functions as shown in Figure 6. 


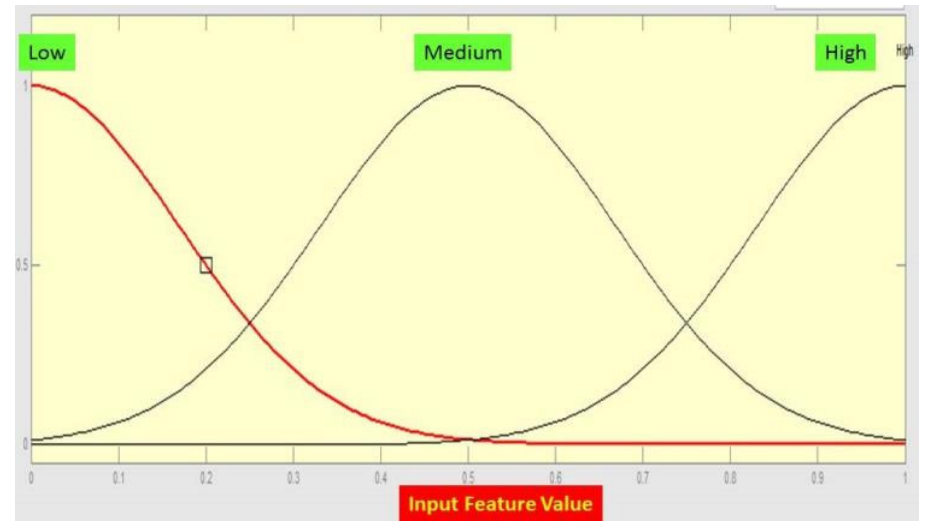

Figure 6. Input feature fuzzification memberships. All the three inputs are fuzzified in the same degrees.

The output classes are also kept quite similar to each other and are shown in Figure 7. All the three classes get some score after each classification is done. The largest value is considered as the ultimate class.

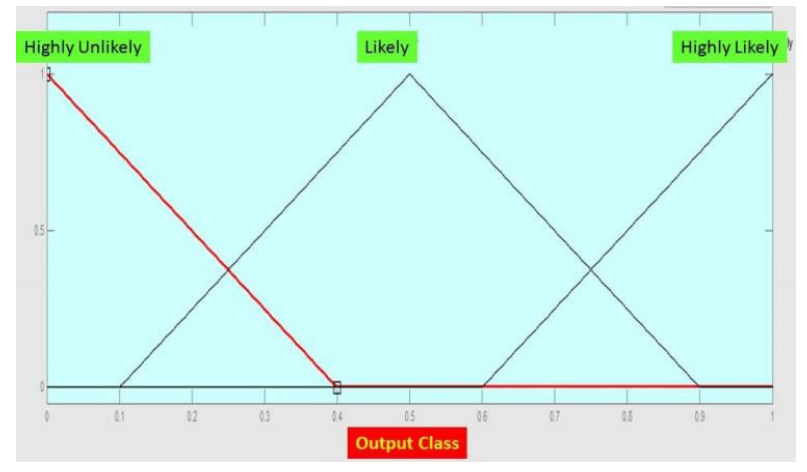

Figure 7. Output classes. All the three outputs have same membership degrees.

The actual mapping from the input to output is done through a set of rules:

1. If (AWR is High) and (GE is Low) and (BE is Low) then (Healthy is HighlyUnlikely)(Corroded is Highly-Unlikely)(Defective is Highly-Likely)

2. If (AWR is Low) and (GE is Low) and (BE is High) then (Healthy is HighlyUnlikely)(Corroded is Highly-Likely)(Defective is Highly-Unlikely)

3. If (AWR is Low) and (GE is Low) and (BE is Low) then (Healthy is HighlyLikely)(Corroded is Highly-Unlikely)(Defective is Highly-Unlikely)

4. If (AWR is Low) and (GE is Medium) and (BE is Low) then (Healthy is HighlyLikely)(Corroded is Highly-Unlikely)(Defective is Highly-Unlikely)

5. If (AWR is Low) and (GE is Medium) and (BE is Medium) then (Healthy is HighlyLikely)(Corroded is Highly-Unlikely)(Defective is Highly-Unlikely)

These rules are translated into decision surfaces which can be used on the fly to classify the health of the sample under study. Figure 8 shows these classes. 


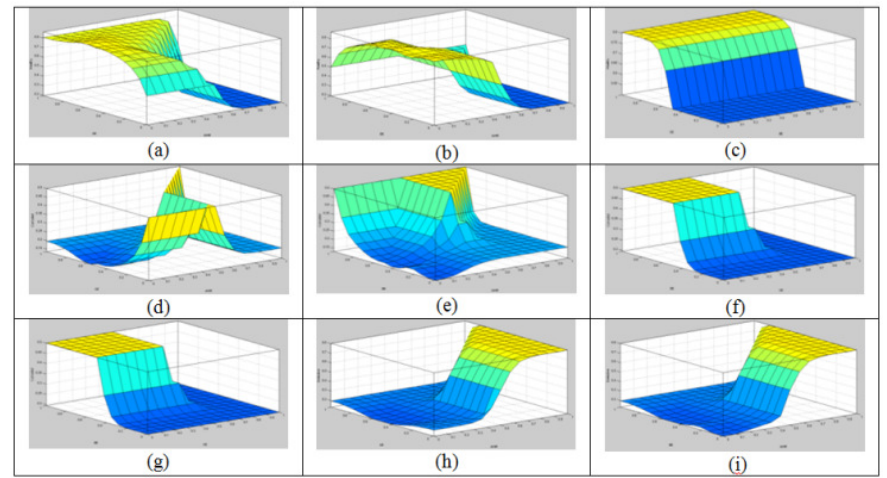

Figure 8. Various decision surfaces. (a) through (c) for Healthy class between the parameters AWR-GE, AWR-BE, and GE-BE. (d) through (f) for Corroded class between the parameters AWR-GE, AWR-BE, and GE-BE. (g) through (i) for Defective class between the parameters AWR-GE, AWR-BE, and GE-BE.

\section{TESTING AND RESUlts}

The algorithm was tested by moving the robot on a $3.5 \mathrm{~m}$ diameter iron plat with a horizontal weld defect in the upper half of the plate. Figure 9 shows the MFL Sensor data received without the location constraints.

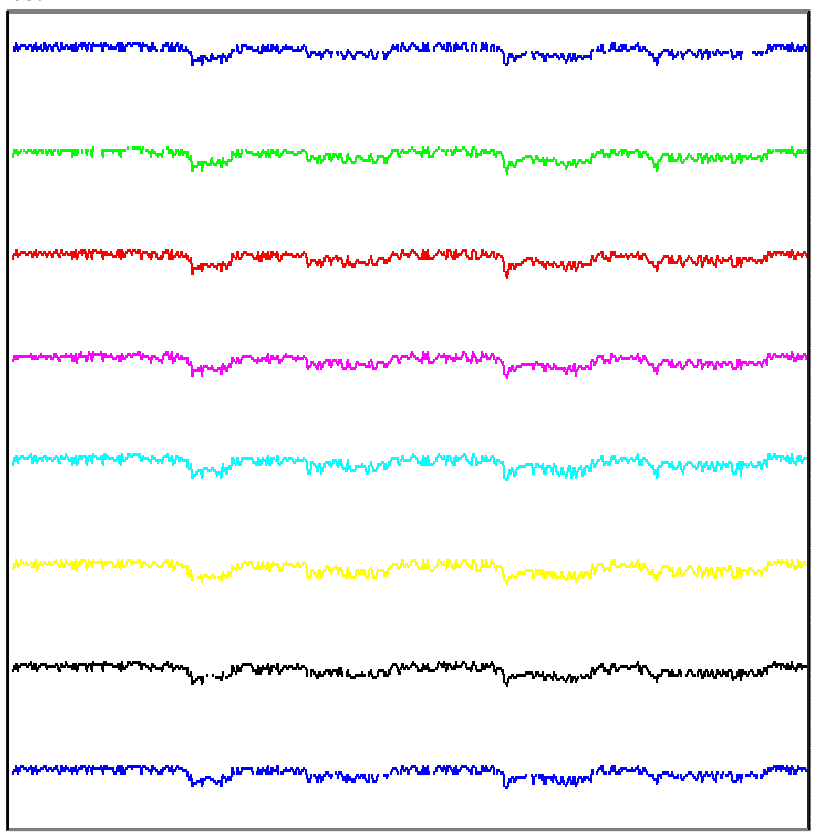

Figure 9. Sensor data from 8 out of 16 sensors in the MFL sensor head.

Once the calculations are done on the data based on the algorithm discussed above, the location is also decided using the laser ranging mechanism of the robot. Figure 10 shows the resulting floor map for this test run. The area shown in the figure is $30 \times 15 \mathrm{~cm} 2$ and clearly shows the three classes in three color levels. The dark brown color represents the weld, the bluish tint represents the corrosion and the orange color represents the healthy metal. Rest of the sample area is kept at dark blue color to indicate the area that was not scanned. 


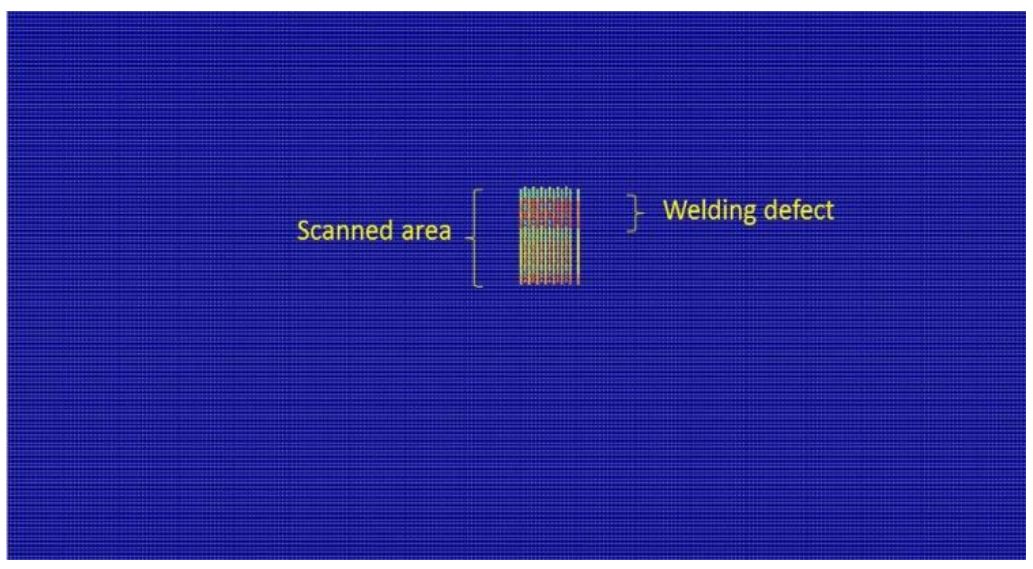

Figure 10. Floor map with Coiflet deconvolution and Fuzzy classification applied.

\section{CONCLUSION}

The Robotic Inspection System developed in this work has shown some very promising outcomes in the testing phase and is expected to improve in terms of its performance and intelligence as more tests will be performed on more realistic defects. The basic sensing mechanism can be used to test the MFL sensing capabilities on a number of test samples and more realistic database can be developed. More realistic samples will provide more insight into how to detect a specific type of defect.

MFL based inspection techniques are quite common in the oil and gas industry. The human dependence in the process limits the accuracy due to the way the MFL data is perceived by the operator of the inspection equipment. The Robotic inspection system presented here is quite superior to these limitations but requires a lot of signal processing to evaluate the signals obtained from the sensors on the robot. The presented technique has shown very promising results with real MFL data and is being tested on industrial targets to build more confidence and accuracy in the inspection procedure.

While several algorithms were tested, the presented algorithms in this report, by far, are the best we have for the purpose of deconvolution and classification. The Fuzzy classifier will enhance with time as newer samples will provide newer features which will make classification more reliable. We really hope that this robotic system will flourish further into an industrial platform that can add to the value of the prime industry in Qatar, the oil \& gas sector.

The raw data waveforms are clearly indicators of the complexity of the data type involved and the need for a better proximity decision related to the inspection. As can be seen from the presented results, the trends are preserved quite well but the unwanted artifacts are significantly reduced, if not removed all together using the deconvolution method.

\section{ACKNOWLEDGEMENTS}

This publication was made possible by a grant from Qatar National Research Fund under its National Priority Research Program, for project NPRP 08-276-2-091. Its contents are solely the responsibility of the authors and do not necessarily represent the official views of Qatar National Research Fund. 


\section{REFERENCES}

[1] The art of Tank gauging, Technical notes, ENRAF Netherlands, 2006.

[2] http://www.ndted.org/AboutNDT/SelectedApplications/TankInspection/TankInspection.htm.

[3] Dury, J.C., and Marino, A., "A Comparison of the Magnetic Flux Leakage and Ultrasonic Methods in the detection and measurement of corrosion pitting in ferrous plate and pipe"www.ndt.net/article/wcndt00/papers/idn701/idn701.htm

[4] Song, Q., "Interacting effects of clustering defects on MFL signals using FEA",

[5] BINDT Insight, Vol 55, No. 10, 558-560, October 2013.

[6] The MFL Compendium: Articles on Magnetic Flux Leakage, ISBN: 978-1-57117-210-5, 2010, American Society of Nondestructive Testing (ASNT).

[7] Kim, D. K., et. Al., "Development of MFL system for in-pipe robot for unpiggable natural gas pipelines", 10th International Conference on Ubiquitous Robots and Ambient Intelligence (URAI), 2013, pp. 51-54.

[8] Kandoori, M. R., Shirani, F., Araabi, B.N. , Ahmadabadi, M.N. , and Bassiri, M.M., "Defect detection and width estimation in natural gas pipelines using MFL signals", 9th IEEE Asian Control Conference (ASCC), 2013, pp. 1-6.

[9] Kikuchi, H., Sato, K., Shimizu, I., Kamada, Y., and Kobayashi, S., "Feasibility Study of Application of MFL to Monitoring of Wall Thinning Under Reinforcing Plates in Nuclear Power Plants", IEEE Transactions on Magnetics, Vol. 47, No. 10, 2011, pp. 3963-3966.

[10] Sun, L. Y., Li, Y. B., Sun, L. B., Li, L. G., "Comparison of Magnetic Flux Leakage (MFL) and Acoustic Emission (AE) techniques in corrosion inspection for pressure pipelines", 31st Chinese Control Conference (CCC), 2012, pp. 5375-5378.

[11] Han, W., and Que, P., "A modified wavelet transform domain adaptive FIR filtering algorithm for removing the SPN contained in the MFL data", IEEE International Conference on Industrial Technology, 2005, pp. 152-157.

[12] Han, W., Xu, J., and Tian, G., "MFL inspection defect reconstruction based on self-learning PSO", Far East Forum on Nondestructive Evaluation/Testing: New Technology \& Application (FENDT), 2013, pp. 50-54.

[13] Han, W., and Que, P., "Defect reconstruction from MFL signals using improved genetic local search algorithm", IEEE International Conference on Industrial Technology, ICIT 2005, pp. 1438-1443.

[14] Lee, J. Y., Afzal, M., Udpa, L., Udpa, S., and Massopust, P., "Hierarchical rule based classification of MFL signals obtained from natural gas pipeline inspection", Proceedings of the IEEE-INNS-ENNS International Joint Conference on Neural Networks, 2000, Vol. 5, pp. 71-78.

[15] Melikhov, Y., Lee, S.J., Jiles, D.C., Lopez, R., and Brasche, L., "Analytical approach for fast computation of magnetic flux leakage due to surface defects", Digests of the IEEE International Magnetics Conference, 2005, (INTERMAG Asia 2005), pp. 1165-1166.

[16] Wei, D., Coiflet type Wavelets: Theory, Design, and Applications, Ph.D. dissertation, University of Texas at Austin, 1998.

[17] MATLAB's link for the Coiflet Wavelet: http://www.mathworks.com/help/wavelet/ug/waveletfamilies-additional-discussion.html.

\section{Authors}

Uvais Qidwai received his $\mathrm{Ph} . \mathrm{D}(\mathrm{EE})$. from the University of Massachusetts-Dartmouth USA in 2001, MS(EE) in 1997 from KFUPM Saudi Arabia, and BS(EE) in 1994 from NED University of Engineering \& Technology, Karachi, Pakistan. He taught in the Electrical Engineering and Computer Science Department, Tulane University, in New Orleans as Assistant Professor, and was in-charge of the Robotics lab as well as a research member of Missile Defence Centre, during June 2001 to June 2005. He joined the Computer Science and Engineering Department, Qatar University, in FALL of 2005

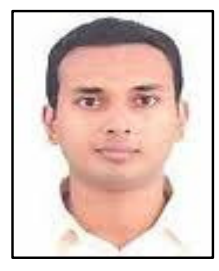
where he is currently working as Associate Professor. Dr. Qidwai's present research interests include Robotics, Signal and Image Processing, Expert Systems design for Industrial Applications, and Intelligent Algorithms for medical informatics. He has participated in several government- and industry-funded projects in the United States, Saudi Arabia, Qatar, UAE, Singapore, Malaysia, and Pakistan, and has published over 100 papers in reputable journals and conference proceedings. 
Mohamed Shakir received the B.Tech degree in Applied Electronics and Instrumentation from MES College of Engineering, Calicut University, India, in 2005 and the M.S. degree in Electrical Engineering from Washington International University, USA in 2011. He is currently pursuing the Ph.D. degree in Electrical Engineering at Univeristi Teknologi Petronas, Malaysia. From 2011 to 2014, he was a Research Assistant with the Computer Engineering Department, Qatar University, Qatar. His research interest is in Robotics.

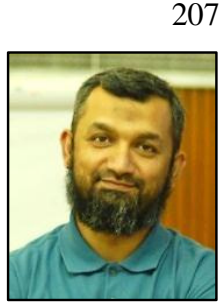

